\title{
Lingual clamping procedures for measuring oral vibrotactile thresholds: IV. Comparison of clamping and nonclamping methods
}

\author{
DONALD FUCCI, LARRY H. SMALL, and LINDA PETROSINO \\ School of Hearing and Speech Sciences, Ohio University, Athens, Ohio 45701
}

\begin{abstract}
Lingual vibrotactile thresholds were obtained from 10 adult subjects using five different clamping procedures at three test frequencies. Data indicated that the elimination of the bottom clamp will lead to thresholds comparable to those involving the use of a bottom clamp, provided that the amount of lingual constriction is carefully monitored. Elimination of the lower clamp is desirable in that it simplifies overall procedure for the experimenter and the subject being tested. Future research relating frequency response characteristics to individual clamping conditions is suggested.
\end{abstract}

Much research involving lingual vibrotactile stimulation has been generated over the last few years with the goal of acquiring a practical tool in assessment of the lingual tactile sensory feedback system. One area of recent concern has been that of clamping the tongue in the test position.

In a study comparing vibrotactile thresholds from the dorsal lingual and palmar surfaces, Verrillo (1968) discovered that the palmar thresholds exhibited a selective "U-shaped" frequency response between 60 and $640 \mathrm{~Hz}$. Conversely, the thresholds from the tongue did not display a selective frequency response. These results conflicted with those of Sherrick (1953), who demonstrated selective frequency responses for both fingertip and tongue tip. The main procedural discrepancy between these two studies is that Sherrick did not use a free-surround disk, which, according to Verrillo, limited the spread of vibratory stimulation to Pacinian receptors present in the ventral portion of the tongue.

Telage, Fucci, and Crary (1977) examined lingual vibrotactile thresholds across five frequencies employing a free-surround disk. Their results indicated that the use of a free-surround clamping disk limits stimulus spread and therefore results in greater threshold sensitivity. Also, their results showed that the lingual tactile receptors are frequency selective, unlike the findings of Verrillo (1968). In a companion study, Fucci, Crary, and Telage (1977) studied the lingual vibrotactile thresholds obtained using a solid lower clamping disk, in hopes of finding a frequency-selective response from the dorsal lingual surface. Their results indicated that when a solid lower clamping disk was utilized (as opposed to a lower ring disk), greater threshold sensitivity occurred. Their findings also support the contention that the lingual dorsal surface demonstrated a frequency-specific response to vibratory stimuli regardless of the clamping procedure utilized. Fucci et al. concluded that the combination of the free-surround disk and solid lower clamping disk provided the most sensitive thresholds.

Telage and Petrosino (1978) examined lingual vibrotactile thresholds using a nonclamping method. They examined thresholds at five frequencies from 60 to $250 \mathrm{~Hz}$ utilizing a free-surround disk, with no lower clamp. Thresholds obtained showed a flat non-frequencyspecific response, which, according to Telage and Petrosino, occurred because clamping "distorted lingual tissue so as to excite more ventrally based energy transducers" (1978, p. 293).

To date, there has been no one study that has focused on the comparative results of clamping vs. nonclamping methodology. Therefore, the purpose of the present investigation is to examine the effects of clamping vs. nonclamping procedures in lingual vibrotactile threshold measurement.

\section{METHOD}

\section{Subjects}

Ten female subjects with no history of speech and/or hearing disorders were randomly selected for this study. All subjects were enrolled at Ohio University. They provided an age range of 21-32 years, with a mean age of 24 years.

\section{Apparatus}

The equipment used in this study has been described in previous publications, the most recent being a chapter on the state of the art (Fucci \& Crary, 1979). The stimulus unit included a sine-wave generator, a frequency counter, an electronic switch-interval timer, an amplifier, a 2-dB step-variable attenuator, and an electromagnetic minivibrator. The pulsed vibratory signal generated had a $50 \%$ duty cycle (on $.5 \mathrm{sec}$ and off $.5 \mathrm{sec}$ ) with a rise and decay time of $100 \mathrm{msec}$. The measurement unit consisted of an accelerometer, a cathode follower, a microphone amplifier, and a voltmeter. A narrowband noise generator was used to present auditory masking at $70 \mathrm{~dB}$ HTL to subjects through TDH-39 headphones.

\section{Procedure}

Threshold measurement was completed under five clamping conditions at $125 \mathrm{~Hz}, 250 \mathrm{~Hz}$, and $500 \mathrm{~Hz}$. In Condition 1, a 
free-surround disk with no bottom clamp was employed. This constituted the nonclamping procedure. Condition 2, utilized both the free-surround disk and a solid lower clamping disk, set 1 in. apart. This condition approximated the clamp placement for threshold measurement as typically executed in previous studies performed at Ohio University. In Condition 3, the bottom clamp was raised into the tongue $1 / 8 \mathrm{in}$. farther than the position in Condition 2. The bottom clamp was raised into the tongue $1 / 8 \mathrm{in}$. farther than the position for Condition 3 to obtain the position for Condition 4 . In Condition 5 , the clamp was raised into the tongue $1 / 8$ in. farther than the position for Condition 4. Clamp adjustment was monitored by use of a ruler attached to the clamping apparatus so that all adjustments were identical across subjects.

Prior to testing, subjects received training to acquaint them with the nature of the stimulus and to familiarize them with the testing apparatus. The subjects were trained to detect the stimulus on the tongue at a frequency of $250 \mathrm{~Hz}$. A training criterion requiring each subject to produce three threshold responses in which no two varied by more than $10 \mathrm{mV}$ was used as an index of adequate training. Subsequent to adequate training, standardized instructions were read to each subject during the testing session. Each subject was seated in an adjustable chair and asked to extend his tongue between the two plastic sterilized disks set 1 in. apart, as described for Condition 2. A hole in the top disk provided access of the probe to the anterior midline section of the dorsum of the tongue. The contactor area of the probe was $.128 \mathrm{~cm}^{2}$. To maintain constant pressure of the vibrator on the tongue for all subjects, the probe was lowered until a voltmeter recorded contact. The probe was then lowered $1 \mathrm{~mm}$ into the tongue surface from the point of recorded contact.

Each subject was required to respond by raising her hand as soon as she detected the pulsed vibratory stimulus. An ascending psychophysical method of limits was used. The mean of three millivolt readings was accepted as the lingual threshold and was later changed to displacement in microns with the standard " $\mathrm{g}$ " formula for acceleration conversion.

Subjects were tested on two separate days. On the 1st day, all subjects were tested at $250 \mathrm{~Hz}$, under all five clamping conditions. On the 2 nd day, they were tested at the frequencies of $125 \mathrm{~Hz}$ and $500 \mathrm{~Hz}$, which were randomly administered under all conditions. This procedure was followed to prevent fatigue. Clamping Conditions 1 and 2 and the combination of Conditions 3,4 , and 5 were presented in random order on both days of testing. Conditions 3,4 , and 5 were grouped together, since clamp position for Condition 4 depended on clamp position for Condition 3 and clamp position for Condition 5 depended on clamp position for Condition 4 .

\section{RESULTS AND DISCUSSION}

Raw data in microns of peak displacement were compiled and averaged for each of the five experimental conditions and for each of the three frequencies tested. Means and standard deviations for the five experimental conditions and for the three frequencies are shown in Tables 1 and 2, respectively. The means for the experimental conditions were similar, except for Treatment 5 , the tightest clamping position. The standard deviations were also similar except for Treatment 5, which displayed a larger standard deviation. The means and standard deviations for all three frequencies varied, with greatest differences noted between $250 \mathrm{~Hz}$ and $500 \mathrm{~Hz}$.

A treatment by treatment by subject analysis of variance was performed to determine if there were any significant differences between the means of the experi-
Table 1

Threshold Means (in Microns) and Standard Deviations for Each Clamping Condition

\begin{tabular}{ccc}
\hline Condition & Mean & SD \\
\hline 1 & 1.75 & .99 \\
2 & 1.77 & 1.10 \\
3 & 1.65 & .97 \\
4 & 1.87 & 1.05 \\
5 & 2.20 & 1.45 \\
\hline
\end{tabular}

Table 2

Threshold Means (in Microns) and Standard Deviations for Each Frequency (in Hertz)

\begin{tabular}{ccc}
\hline Frequency & Mean & SD \\
\hline 125 & 1.80 & .88 \\
250 & 1.36 & .48 \\
500 & 2.40 & 1.53 \\
\hline
\end{tabular}

Table 3

Summary Table for the Analysis of Variance

\begin{tabular}{lrrrc}
\hline \multicolumn{1}{c}{ Source } & \multicolumn{1}{c}{ SS } & df & MS & F \\
\hline Subjects & 81.81 & 9 & 9.09 & \\
Condition & 5.51 & 4 & 1.38 & $6.31^{*}$ \\
$\quad$ Error & 7.85 & 36 & .22 & \\
Frequency & 26.51 & 2 & 13.26 & $4.32 \dagger$ \\
$\quad$ Error & 55.21 & 18 & 3.07 & \\
Condition by Frequency & 1.05 & 8 & .13 & .82 \\
$\quad$ Error & 11.64 & 72 & .16 & \\
Total & 189.58 & 149 & & \\
\hline
\end{tabular}

${ }^{*} p<.01 . \quad \dagger p<.05$.

mental conditions and those of the frequencies under examination. The $\mathrm{F}$ ratio obtained for the experimental conditions $(6.31, \mathrm{df}=4,36)$ was significant at the .01 alpha level. Similarly, the $F$ ratio obtained for the three frequencies $(4.32, \mathrm{df}=2,18)$ was significant at the .05 alpha level. However, the $F$ ratio obtained for the interaction between conditions and frequencies $(.82, \mathrm{df}=$ 8,72 ) was not significant at the .05 level. These results are summarized in Table 3.

A Tukey HSD test was performed to determine where the statistically significant differences existed with respect to the five clamping conditions. A significant difference at the .01 alpha level was determined to exist between Condition 5 and Conditions 1,2 , and 3 $[\mathrm{r}(36)=5, \mathrm{q}=.42]$. A second Tukey HSD test was performed on the three frequency conditions. Statistical significance at the .05 alpha level was found between $250 \mathrm{~Hz}$ and $500 \mathrm{~Hz}[\mathrm{r}(18)=3, \mathrm{q}=.89]$.

It appears from the data collected that lingual vibrotactile threshold measurement can be obtained with similar results whether or not a solid lower clamping disk is used. It should be noted that once the lower clamping disk is raised $3 / 8$ in. into the tongue, statistically significant threshold results do occur. Therefore, if clamping is still desired in obtaining thresholds, the lower clamp should not be set greater than $2 / 8$ in. into 
the ventral portion of the tongue. It is of interest to note that a number of the subjects remarked about how much more comfortable and how much easier the task was when the bottom clamp was removed completely. According to Fucci et al. (1977), the combination of an upper and lower clamping disk provided the most sensitive thresholds. Although they tested subjects with the upper clamp removed (as opposed to the present study, which tested subjects with the lower clamp removed), Fucci et al. stated that both clamps were necessary for stable positioning of the tongue. According to results of the present study, this does not appear to be the case. In the study by Telage and Petrosino (1978), it was stated that "lingual clamping may distort tissues so as to excite ventrally based frequencyresponsive mechanoreceptors" (p. 291). This appears to be so, once clamping is increased beyond $2 / 8$ in. into the ventral aspect of the tongue.

In examination of the mean frequency data for all clamping conditions combined, the present results reflect the differential frequency response of the tongue often reported in the literature (Telage \& Fucci, 1973, 1974a, 1974b). The thresholds indicated greatest sensitivity at $250 \mathrm{~Hz}$, which is within the range of frequencies to which the tongue is most sensitive (Fucci, Hall, \& Weiner, 1971).

It appears from the findings of the present study that the use or nonuse of a lower clamp to position the tongue for vibrotactile testing is of no consequence. If a lower clamp is employed, however, the degree of tightening with respect to the lower tongue surface should be carefully monitored. If the lower clamp is eliminated, this careful monitoring is not necessary and the entire procedure is simplified for both the experimenter and the subject being tested.

The original intent of this study was to examine the effects of clamping vs. nonclamping procedures in lingual vibrotactile threshold measurement. However, the present data also suggest that there is a need for investigation of the frequency response characteristics of the tongue as they relate to the individual clamping conditions. Future research endeavors in this direction will lead to further methodological clarification necessary for accurate lingual vibrotactile threshold assessment.

\section{REFERENCES}

Fucci, D., \& Crary, M. Oral vibrotactile sensation and perception. New York: Academic Press, 1979.

Fucci, D., Crary, M. A., \& Telage, K. M. Lingual clamping procedures for measuring oral vibrotactile thresholds: II. Effects of using a free clamping disk. Bulletin of the Psychonomic Society, 1977, 10, 457-459.

Fucci, D., Hall, D., \& Weiner, F. Normative study of oral and nonoral structures using vibrotactile stimuli. Perceptual and Motor Skills, 1971, 33, 1099-1105.

Sherrick, C. E. Variables affecting sensitivity of the skin to mechanical vibration. Journal of Experimental Psychology, 1953, 45, 273-282.

Telage, K. M., \& Fucci, D. J. Vibrotactile stimulation: A future clinical tool for speech pathologists. Journal of Speech and Hearing Disorders, 1973, 38, 442-447.

Telage, K. M., \& Fucci, D. J. Concerning intrasubject measurements of successive lingual vibrotactile responses. Perceptual and Motor Skills, 1974, 39, 1047-1052. (a)

Telage, K. M., \& Fucci, D. J. Measurement of lingual vibrotactile threshold sensitivity using one-trial and three-trial threshold criteria. Bulletin of the Psychonomic Society, 1974, 3, 373-374. (b)

Telage, K. M., Fucci, D. J., \& Crary, M. A. Lingual clamping procedures for measuring oral vibrotactile thresholds: I. Effects of using a free-surround disk. Bulletin of the Psychonomic Society, 1977, 10, 347-349.

Telage, K. M., \& Petrosino, L. A. Testing procedures for measuring oral vibrotactile thresholds: III. Effects obtained using a nonclamping method. Bulletin of the Psychonomic Society, 1978, 12, 291-293.

VERRILLO, R. T. A duplex theory of mechanoreception. In D. R. Kenshalo (Ed.), The skin senses. Springfield, Ill: Thomas, 1968.

(Received for publication August 20, 1981.) 